\title{
EL RECONOCIMIENTO: UNA CONDICIÓN INDISPENSABLE PARA LA CONSOLIDACIÓN DEL ESTADO
}

\author{
Armando Lugo GonzÁlez* \\ RAFAEL JimÉnEZ VEGA**
}

\section{Resumen}

Al realizar un análisis de las teorías positivistas se aprecia que se deja de lado que los seres humanos son individuos de carencias, y por ello se asocian voluntariamente con base en el compromiso moral que hace posible la convivencia. Para desarrollar este postulado se ausculta la teoría del Estado de Tomas Hobbes y se hace un breve esbozo de otras como la de Max Weber, para determinar que en ellas se soslaya que el ser humano, al depender de otros, ha generado mecanismos para facilitar la vida en comunidad que van más allá de la naturaleza egoísta y violenta que requiere ser disciplinada. Por ello se hace especial referencia a las emociones y los sentimientos que motivan al ser humano a tener un comportamiento moral, para lo cual es necesario una acción de reconocimiento de la otredad y, con base en ello, construir la convivencia, a fin de consolidar el Estado.

Palabras clave: Estado, reconocimiento, reificación, teoría, emociones, sentimientos.

\section{RECOGNITION AS A CONDITION FOR THE STATE CONSOLIDATION}

\begin{abstract}
This article builds on a critical view to positivist theories. We argue that the fact that human beings are beings of deprivation has been overlooked as a driver of voluntarily association that stands on the moral commitment to
\end{abstract}

* Estudiante del Doctorado en Estudios Políticos de la Universidad Externado de Colombia (Colombia). Docente, Universidad Distrital "Francisco José de Caldas", Bogotá (Colombia). [arlugog@gmail.com].

** Doctor en Estudios Políticos, Universidad Externado de Colombia (Colombia). Docente de Cátedra, Universidad Escuela de Administración de Negocios EAn y Universidad Sergio Arboleda. [rafaeljimenezv68@hotmail.com]. Recibido: 15 de julio de 2017 / Modificado: 14 de febrero de 2018 / Aceptado: 20 de febrero de 2018.

Para citar este artículo

Lugo González, A. y Jiménez Vega, R. (2018). El reconocimiento: una condición indispensable para la consolidación del Estado. OPERA, 22, pp. 141-161.

DoI: https://doi.org/10.18601/16578651.n22.08 
good behavior that makes coexistence possible. In order to develop this postulate we discuss Tomas Hobbes' theory of the State and briefly sketch other theories. The argument follows the idea that human beings, depending on other people, have generated mechanisms to facilitate life in community, reaching beyond the selfish and violent nature that requiring discipline. We point to emotions and feelings as drivers of moral behavior. These require recognition of otherness and coexistence and may poausibly be a core State foundation.

Key words: State, recognition, reification, theory, emotions, feelings.

Al realizar un análisis de las teorías positivistas del Estado se observa que su visión es incompleta, como es el caso de la formulada por Tomas Hobbes, al pretender explicar que este surge para mitigar la acción violenta natural de las personas y así hacer posible la convivencia; por esta misma línea se orienta la idea del Estado fuerte aportada por Max Weber, la cual deja de lado que el ser humano, al depender de sus congéneres, ha generado mecanismos para facilitar la convivencia ${ }^{1}$ que van más allá de la naturaleza egoísta y violenta que requiere ser disciplinada. Por ello se considera que, como complemento a las teorías predominantes, se debe hacer especial referencia a las emociones y los sentimientos que motivan al ser humano a tener un comportamiento moral ${ }^{2}$, porque la compasión ${ }^{3}$ y el buen actuar han hecho parte de la construcción social del Estado, creando unas normas que al tener presente al otro regulan la conducta de los individuos. Esto se puede interpretar como la necesidad de reconocimiento como fundamento de la convivencia, siendo esta el pilar en que se consolida el Estado.

Para lograr esto se aborda un marco teórico que, en primera instancia, acoge los conceptos de Adela Cortina, que ayudan a entender que la vida ética ${ }^{4}$ del reconocimiento recíproco es el fundamento que construye las sociedades; en este ámbito son determinantes las emociones que inhiben la mutua instrumentalización de las personas, lo que lleva a respetar la autonomía ajena y la propia. Siguiendo por este conducto, de la mano de Martha Nussbaum, la reflexión se orientará a entender que las sociedades se organizan y se determinan con base en

1 La convivencia está conformada por la seguridad, tranquilidad, moralidad y medio ambiente o ecología (Londońo, 2011, p. 17 ).

2 Adela Cortina (2009, pp. 44-45) aclara que los vocablos "moral" y "ética" significan practicamente lo mismo desde el punto de vista etimológico, costumbres, carácter, usos. El nivel de la vida cotidiana es en el que la gente vive su vida moral, porque no hay personas amorales, se nutren para ello de tradiciones religiosas o seculares de la vida corriente; el nivel de la filosofía moral o ética trata de aclarar en qué consiste ese fenómeno de la vida moral, por qué lo hay y cómo se aplican los principios en las distintas esferas de esa vida corriente. De esta manera, se asigna el término "moral" a la vida cotidiana y el término "ética" a la filosofía moral como una convención.

3 Entiéndase por compasión el sentimiento que despierta el sufrimiento de otra persona, el cual motiva el actuar para aliviar ese padecimiento.

4 Para Adela Cortina (2009, p. 21), la ética intenta descubrir los procedimientos racionales que permiten tomar decisiones sobre la justicia de las normas, descubrir los procedimientos racionales necesarios para decidir conjuntamente qué es lo más justo, y deja a las personas afectadas decidir qué consideran adecuado. 
su repertorio emocional. Finalmente se concluye con los planteamientos realizados por Axel Honneth, para quien la relación del hombre consigo mismo y con el mundo parte de una acción de reconocimiento que conduce a una actitud de apoyo antes de otras orientaciones.

Por consiguiente, se consultarán diversas fuentes comenzando por El Leviatán de Hobbes, para posteriormente auscultar los diferentes aportes que han cuestionando las teorías positivistas, al encontrar que por su inflexibilidad no permiten tener en cuenta otros factores como el reconocimiento, la vida ética o las emociones, para de esta forma, apoyados en autores normativos, concluir que el reconocimiento es un factor indispensable para la consolidación del Estado.

Se puede comenzar por entender que las emociones inducen a los comportamientos morales; se define por moral "el conjunto de normas y creencias de primer orden relativas a la consecución de una vida buena y justa, así como a las prácticas que supone el seguimiento de dichas normas" (Villoria e Izquierdo, 2016, p. 16). Esto genera interrogantes como: ¡es pertinente una crítica al positivismo hobbsiano al no tener en cuenta el reconocimiento en su concepto sobre el Estado? ¿Se debe realizar una crítica a otras teorías positivistas del Estado que no incluyen el concepto del reconocimiento? ¿Existen razones por las cuales el Estado requiera del reconocimiento para su consolidación? ¿Qué importancia tienen las emociones y los sentimientos en la consolidación del Estado? ¿El reconocimiento es un factor relevante en la consolidación del Estado?

El tener en cuenta al ser humano en su esencia nos lleva a decir que el Estado es más que "la máxima organización de un grupo de individuos sobre un territorio en virtud de un poder de mando" que adujo Maquiavelo en su momento (Bobbio, 1989, p. 86). Esta indagación pretende auscultar algunas teorías positivistas del Estado con las que se han pretendido explicar las diferencias y la forma como se han ido organizando estructuralmente las colectividades humanas, en donde el Estado es visto como el ente que entra a controlar la realidad espontánea de la sociedad. Se debe tener en cuenta que la conformación del Estado ha estado acompañada de los procesos sociales, porque este no es una figura vacía, sino un marco de acción colectiva, como lo enuncia Chevallier (2011, p. 41), que a su vez ha estado inmerso en una constante evolución de los factores políticos y económicos, entre los cuales destaca Vela (2010, p. 37): la ciencia natural y el consecuente desarrollo tecnológico; el capitalismo desde sus inicios mercantilistas e industriales; el absolutismo y la centralización del poder; la reforma protestante y la secularización entre lo político y lo religioso que pasan a darle forma al Estado moderno.

De acuerdo con esto se puede colegir que la nación se consolida a través del Estado como un proceso del sentir social dentro de un marco de libertad e igualdad. Esta evolución acerca de lo público y lo privado, como pretendía Hannah Arendt, cambia en definitiva el curso de la reflexión secular de la relación entre Estado y sociedad que ya no es distante, sino que el primero es consecuencia del segundo, entendiendo que el afán por la seguridad conduce a las personas a llegar a acuerdos que preceden a los sentimientos morales e implican una necesidad de reconocimiento. 


\section{CRÍTICA AL POSITIVISMO HOBBSIANO POR AUSENCIA DEL CONCEPTO DE RECONOCIMIENTO}

Ahora se procede a plantear una crítica al modelo del Estado de Hobbes a partir de los postulados de Adela Cortina, para así hacer visibles las limitaciones e insuficiencias que se desprenden desde esa óptica positivista contractualista e individualista. Para ello, primero se enuncia el valioso aporte de Baruch Spinoza que critica la visión hobbsiana, para después hacer una breve exposición del desarrollo del concepto contractualista y, de esta manera, poder soportar que, evidentemente, el compromiso moral a la norma y la interdependencia de los seres humanos crea vínculos emocionales que cohesionan la sociedad y, por su conducto, al Estado.

En la modernidad, cuando se comienza a dar importancia a lo terrenal, asimismo se busca encontrar la razón por la cual el ser humano, en vista de su dependencia, resuelve dar uso al regalo que Prometeo le entregó para facilitar su vida en comunidad, cual fue la política, el uso de la razón expresado a través del diálogo para asíllegar a acuerdos que le permitieran obtener para sí y sus seres queridos la tranquilidad y seguridad necesarias.

Desde Platón, los filósofos han soñado con una élite de gobernantes poseedores de dones exclusivos que les permitan dirigir con base en la razón, para asegurar la estabilidad del Estado, controlando ese cuerpo del Leviatán rebelde e ignorante de la muchedumbre. Esa era la supuesta arma política que justificaba su presencia en el Gobierno. Sería Spinoza, con su Tratado teológico-político (1986) y, posteriormente, con el Tratado político (1986), de los primeros en cuestionar la teoría de Hobbes, asintiendo que es el miedo el que la mayoría de veces lleva al hombre a unirse, aunque cuestiona que esta sea la única causa, pues no hay un poder omnipotente sobre las pasiones que explique su naturaleza y la conducta por la razón. Por consiguiente, las causas y los fundamentos naturales del Estado no deben buscarse en las enseñanzas de la razón, sino en la naturaleza y en la condición común de los hombres. No hay figuras mesiánicas, la naturaleza para todos los hombres es la misma, no hay diferencia de los gobernantes con los gobernados. Las virtudes y la conducta racional de las personas es producto de la vida social y no del origen, el Estado debe obedecer a una organización jurídica común que no esté al arbitrio de los gobernantes, la obediencia civil no se debe a un sometimiento de ideas, virtudes $\mathrm{u}$ opiniones de ningún particular. $\mathrm{El}$ poder del Estado no es trascendente a los individuos, sino que estriba en la naturaleza misma de las relaciones que ellos mantienen entre sí. El Estado es una organización colectiva que nace por una necesidad vital, cuya existencia requiere de la ayuda recíproca de los hombres para conservar su existencia. La finalidad de las leyes y de las instituciones reside en canalizar esa ayuda, evitando que estalle el conflicto y la lucha provocada por las pasiones opuestas (Allendesalazar, 1988, pp. 89-101).

A estos aportes le anteceden los entregados por los integrantes de la Escuela de Salamanca como Francisco Suárez y Francisco de Vitoria, quienes fueran los que, en primera instancia, hablaran del principio contractualista en la construcción del Estado; les seguirían Johanes Althusius, Jean Bodin y Nicolás Maquiavelo, quienes realizaron un análisis de 
las formas de gobierno y del comportamiento ideal que debería tener el monarca para consolidar su reino en un Estado. Pero sería Thomas Hobbes (1980, pp. 254-262) quien en el capítulo xxvin, de De las Penas y de las Recompensas, mostraría la naturaleza del hombre cuyo orgullo y otras pasiones lo llevan a tener la necesidad de someterse a un gobierno y al gran poder del gobernante, y crea así, a modo de representación de estas ideas sobre el Estado, una figura a la que se le da el nombre de Leviatán. Es el soberano de la arrogancia, hecho para no sentir el miedo, y es el rey de todas las criaturas soberbias, nada existe sobre la tierra que pueda compararse con él, pero destaca que es mortal como todas las criaturas de la tierra y por eso siente temor, por lo que debe obedecer unas leyes.

Esta imagen o frontispicio contiene una cita en latín que se extracta de Job 41,24: Non est potestas super terran quae comparetur (no hay poder sobre la tierra que pueda compararse a él), se destaca una figura humana gigante con su cabeza coronada que sostiene en su mano derecha la espada que representa el poder terrenal o temporal y en la izquierda un báculo pastoral que representa el poder espiritual o eclesiástico; su torso está formado por una multitud de pequeñas figuras humanas que ejemplifican el pacto que hacen creando una sola y misma persona; en un primer plano, cubriendo el resto del cuerpo del gigante, se observa un paisaje de colinas en el que se encuentran varios pueblos y lleva a desembocar en una imagen de la ciudad en la que se reconoce en la parte izquierda que corresponde al báculo, una catedral, y en la margen derecha, que corresponde a la espada, una fortaleza; una especie de marco divide en la parte inferior y superior la imagen, continúan unas figuras que van en correspondencia con el poder que representa cada brazo del gigante, son unos emblemas (cinco de cada lado) que se refieren, en el lado derecho, al poder temporal (fortaleza, corona, cañón, una panoplia de estandartes y una batalla), por el lado, al eclesiástico o poder espiritual (iglesia, mitra, el rayo de la excomunión, los símbolos de los silogismos lógicos y una especie de concilio), y, en medio de ellos se encuentra un telón con el título del libro.

Es una indagación que devela la necesidad de ponerle controles a la naturaleza humana por su ambición desmedida, destacando entre las pasiones que más afectan la convivencia: la competencia, la desconfianza y la gloria. La competencia impulsa a los hombres a atacarse mutuamente para alcanzar un beneficio; la desconfianza los lleva a ejercer la violencia para lograr seguridad y defender a su prole; el anhelo de gloria motiva a los hombres a obtener reputación, lo que implica muchas veces también recurrir a la violencia. Se puede inferir en estos casos que, igualmente, para la obtención de cada una se requiere de los demás seres humanos, por ser objeto de carencias y por ello dependientes. Pero para que las pasiones no desborden en conflicto permanente y se puedan alcanzar unos mínimos de seguridad acudiendo a la razón es conveniente sellar un pacto.

Esta disertación sobre las pasiones la desarrolla Hobbes en el capítulo vi (1980, pp. 40-50), en donde también identifica que el temor a la muerte y el deseo de adquirir las cosas necesarias para una vida confortable con la esperanza de obtenerlas por medio del trabajo, no se superan ni se logran con la promoción de la guerra sino a través de acuerdos. Esto es algo que incluso está determinado por la natu- 
raleza, que indica que la comunidad política es el único medio que les garantiza que todos sacrifican su derecho a poseerlo todo, resultando más conveniente acogerse a la ley para alcanzar la paz. Esta decisión es distante de cualquier principio moral, es simplemente una acción de conveniencia para lograr sus objetivos.

Es particular la diferencia que Hobbes establece entre pueblo y multitud, el primero es para él un grupo de personas orientado por la razón, que posee una sola voluntad, por lo que tendría una acción propia y está conformado por la asamblea; por otra parte están los ciudadanos o súbditos que, por ignorantes, son manipulables, por tanto no poseen acción propia, no tienen un significado político y por ello son representados de manera indirecta por la asamblea. Esto lleva a colegir que el soberano es el pueblo que se unifica en la persona del rey, y entrega por consiguiente el poder y su soberanía al monarca.

Giorgio Agamben (2017, pp. 35-65) considera que el pensamiento de Hobbes ha marcado la tradición política occidental creando una escisión entre pueblo y multitud que impide integrarse en un todo. Por consiguiente, se ha interpretado que la multitud está presente salvo a través de las personas que la representan, como ocurrió desde ese primer momento constitucional que significó la Revolución francesa, lo cual ocasiona que esta ausencia de reconocimiento sea la causa de la amenaza de guerra civil permanente.
Vemos ahora que la solución hobbesiana condicionará el desarrollo del pensamiento político moderno, al considerar que el único modo de expresar la voluntad del cuerpo común es la representativa; es decir, a través de uno o de algunos que, en cuanto personas realmente existentes, expresen para todos la voluntad del sujeto colectivo (Duso, 2005, p. 94). Previo a la obra de Hobbes, Johannes Althusius había argüido que el Estado existe por naturaleza y es anterior a cualquier individuo, lo que implicaba que la libertad de los ciudadanos, y no el poder de los gobernantes, era lo que debía primar; a esto se le sumaba el bienestar, la prosperidad y la felicidad de cada individuo, siendo estos los méritos por los que debía caracterizarse un Gobierno, y no por la potencia del Estado. De esta manera, el accionar administrativo se centraba en la cantidad de derechos de que gozaba cada individuo (Bobbio, 1989, p. 84).

Sin embargo, destaca Hobbes en el capítulo xxx (1980, pp. 275-291), que el soberano está obligado a obedecer unas leyes como la de brindar seguridad al pueblo, entendiendo por seguridad no solo la conservación de la vida, sino también todas las excelencias que el hombre pueda adquirir para sí mismo por medio de la actividad legal; cada soberano debe velar por la seguridad de su pueblo como lo haría una persona por su cuerpo, una vida posiblemente feliz, esto es un giro relevante hacia la biopolítica ${ }^{5}$.

5 Este es un término estudiado por Foucault (2016) en el que la sociedad representa el principio en cuyo nombre el gobierno liberal tiende a autolimitarse; de igual manera interviene permanentemente, no para limitar, sino para garantizar y multiplicar las libertades. La sociedad, por su parte, representa el conjunto de las condiciones del menor gobierno liberal porque se autorregula. 
Lo anterior lleva a pensar que a los seres humanos como seres sociales interdependientes, aunque sin sentido moral, les es conveniente obedecer las normas para no perder la vida, la propiedad y su seguridad, además que este ambiente organizado les permite obtener los bienes que anhelan por medio de su trabajo. Es esta también la razón por la cual surge el control social y el rechazo a las conductas anonímicas ${ }^{6}$.

Es por ello que Hobbes determina que existen cuatro razones por las cuales los ciudadanos obedecen las leyes sin excepciones respetando el pacto: la primera es por el estado de naturaleza, que impide la convivencia al hacerse intolerable; la mezquindad y el egoísmo presentes en el no cumplimiento de las normas ponen en peligro la subsistencia de la comunidad política; en segunda instancia, estas circunstancias llevan a las personas a optar por su sometimiento a un gobierno y a obedecer las leyes del soberano; como tercer punto, dadas las condiciones, todos los ciudadanos aceptan de mutuo acuerdo el someterse al gobierno; y como cuarto punto se llega a entender que para el soberano, al ser el representante del ciudadano, cada acto se entiende como acto del ciudadano y por tanto es incoherente que se objete contra lo que hace la misma persona; en consecuencia, el ciudadano ha de obedecer la ley, pero el soberano no está en la obligación de hacerlo porque es su autor. Esto es una consecuencia del principio de representación en que el súbdito no es el que crea la ley y, por ello, no puede cambiarla; por el contrario, el soberano no queda sujeto a ella y está a su arbitrio cumplirla o cambiarla.

En el análisis que hace de la obra de Hobbes, Adela Cortina (2009, pp. 56-75) acota que ese egoísmo inteligente es el resorte que mueve a las personas a cumplir las leyes legales pero no sirve para adquirir convicciones morales. Para ello se tiene que hacer de la obligación legal también una obligación moral, no solo porque hay peligro de sanción, sino porque es justo y conviene a la generalidad. De esta forma aparecen razones diferentes al miedo que conducen al buen obrar, al valor de la justicia y de las personas; sobresale aquí el sentido de la dignidad. Se arguye, incluso, que dentro del comportamiento moral está incluido el reconocimiento jurídico, cada ser humano es un portador de derechos y obligaciones, el reconocer la norma es una afirmación personal y un reconocimiento del otro al considerar que su inobservancia afecta a los dos por igual. Es un proceso de estima social en el que se impone la vida ética ${ }^{7}$ que termina por cobijar a todo el conglomerado, porque no se trata solo del cumplimiento de la norma positivada, sino que pasa a ser más relevante el cumplimiento de la norma informal que indica el buen ac-

6 La anomia es un concepto perteneciente a la tradición teórica de la sociología que significa la ausencia de normas, la tendencia transgresora de las reglas.

7 La ética se compone de un conjunto de juicios y de reglas que sirven para orientar nuestro comportamiento en la vida. Se comporta como una autoridad interna por la que regulamos nuestros actos y juzgamos tanto nuestra conducta como la de los demás. La brújula de la moral nos ayuda a distinguir qué tipo de vida y qué tipo de acciones debemos escoger (Villoria e Izquierdo, 2016, p. 15). 
tuar, que incluso determina el desarrollo de las naciones como bien lo ha establecido Douglass North (1993).

El Estado de Hobbes funciona con un poder absoluto, dice Adela Cortina (2009, p. $70)$, pero este poder es solo exterior, no convence desde dentro, no vincula a la persona consigo misma y con otras, por eso es incapaz de generar alguna obligación moral. No es solo el cálculo inteligente de la supervivencia o el bienestar personal y el de los seres queridos que lleva a obrar a los seres humanos, sino también el aprecio por lo valioso, el disfrute de la relación mutua, la alegría de compartir el gozo, la tristeza por el sufrimiento, la indignación por el daño injusto, la estima de la dignidad ajena y propia.

Por el contrario, Hobbes se fundamenta en el individualismo posesivo que ha descrito MacPherson (2005), en el que se entiende que el núcleo de la vida social es el individuo, en el que cada uno es dueño de sus facultades y su producto, sin deber por ello nada a la sociedad. Cada individuo está convencido de que sus bienes económicos y políticos, los honores que recibe y las riquezas sociales de que dispone son suyos en exclusiva, sin que la sociedad tenga en ello merito alguno. En consecuencia, para Adela Cortina (2009, pp. 71-75), de este individualismo se derivan: el principio supremo de la vida corriente que ella bautiza como Principio del intercambio infinito, el cual se fundamenta en que los seres humanos son seres de carencias, por ello necesitan de otras personas y de lo que el entorno medioambiental pueda ofrecer, el intercambio y la cooperación son más inteligentes que la fuerza bruta. Por eso, en las relaciones sociales se contempla el cálculo de qué podemos obtener de ellas y qué debemos ofrecer a cambio; por otro lado está el Principio Mateo, que consiste en que al que más tiene más se le dará, y al que tiene poco hasta lo poco que tiene se le quitará, con lo que el ser humano queda expuesto y desprotegido ante la injusticia. Es por ello que Cortina sugiere que para romper con el círculo vicioso hay que acudir a la lógica de la cooperación, que incluye los sentimientos sociales.

Todo esto nos lleva a comprender que el primer gran desarrollo de una teoría del Estado, como fue la de Hobbes, fue un argumento al realismo político, que explica claramente cómo las pasiones individuales sin control conducen al estado de guerra permanente de todos contra todos, por lo cual se debe acudir a la razón que induce a ceder en la voluntad para alcanzar un acuerdo en el que las personas aceptan ser gobernadas y a obedecer la ley; esta obediencia pasa a ser el fundamento de la convivencia, y el Estado a ser el garante de una seguridad que no solo defiende la propiedad e impide las invasiones, sino que el orden que impone facilita al hombre alcanzar la felicidad.

Por lo acotado se puede inferir que el egoísmo inteligente induce a obedecer para alcanzar un bien particular, no por un principio moral, creando un vacío que pasa a llenar el lenguaje normativo, porque la condición de seres humanos dependientes obliga a un intercambio permanente; este contacto implica un desarrollo de emociones que conduce a empatías y apatías, el cual lleva a identificarse con lo experimentado y actuado por los semejantes. De este modo, el buen actuar deja de ser egoísta y exterior, ahora surge un compromiso que nace desde el interior, un verdadero anhelo 
por el bienestar de los demás y un rechazo a la anomia porque ella perjudica a la generalidad.

\section{CRÍTICA A OTRAS TEORÍAS POSITIVISTAS DEL ESTADO QUE NO INCLUYEN EL CONCEPTO DEL RECONOCIMIENTO}

Es necesario citar algunas de las teorías del Estado que han obviado el reconocimiento como un elemento esencial para su consolidación; de esta manera se hace evidente que el soslayar el actuar moral y las emociones de las personas tiende a la reificación ${ }^{8}$, así como el subordinar la sociedad al derecho o explicar la formación del Estado como consecuencia de los procesos bélicos.

No se pueden dejar de lado las conjeturas a que han llegado autores como Charles Tilly (1992), quien manifiesta que el origen y desarrollo del Estado-nación está asociado con la coerción y con la guerra, tal como se puede apreciar en Europa occidental, en donde el monopolio de la violencia y los procesos de acumulación capitalista están claramente asociados a su organización y conformación; a esto también habría que adicionarle que la acción bélica es producto de un deseo de reconocimiento de un grupo de personas, ya sea por sus ideas, por sus creencias o simplemente para que se avale su existencia.
Lo que se aplicaría de igual manera a los aportes de Immanuel Wallerstein (2006) en el ámbito de la política internacional, al aducir que las victorias político-militares fueron las que aumentaron de forma crítica las distancias económicas, e influyeron en el desarrollo de la agricultura, la industria, el comercio y las finanzas, fijando así a la violencia como parte de la evolución de lo político, en donde se explota y se es explotado para alcanzar las cotas económicas anheladas que determinan el sistema mundo. Se obvia en esta construcción académica, que estas guerras representan el clamor de los Estados por su reconocimiento y la aceptación de su participación en el escenario internacional, e implica incluso el anhelo de algunos de que sea aceptada una supuesta superioridad sobre los otros.

Se debe entender que esa violencia, a la que también se refiere Vela (2011, p. 72), producida por la razón de Estado para organizar bajo su potestad la vida en común, pasa a depender de igual forma de los sentimientos y las emotividades de las personas para la consolidación de las relaciones de poder estables y legítimas que creen un nuevo orden.

Por su parte, la teoría clásica tradicional y ortodoxa de Max Weber (1964, pp. 170174) dice que el Estado es una organización burocrática, racional y unitaria, con el predominio del poder de la rama ejecutiva en su

8 Reificación es un término que Georg Lukács comenzó a utilizar en su colección de ensayos Historia y conciencia de clase en 1925 (1969, pp. 89-266); hace referencia a un hábito o a la costumbre de una conducta simplemente observadora, desde cuya perspectiva el entorno natural, el entorno social y los potenciales propios de la personalidad son concebidos de manera indolente y desapasionada, como algo que tiene calidad de cosa (Honneth, 2007, p. 30). 
organización burocrática. Una representación de la dominación racional que se opone a la dominación tradicional y carismática explicada por Weber' ${ }^{9}$, fundamentándose en el valor de la previsibilidad como resultado de la confianza en la legalidad, dando a entender que todo ejercicio de autoridad es un ejercicio de administración. Lina Buchely (2014, p. 19) caracteriza esta dominación racional propuesta por Weber en tres premisas fundamentales: 1) la creencia en la validez de la ley como guía de acción; 2) el esquema de competencias creadas mediante normas racionales como una cadena de sucesión de validez formal; 3) la disposición irrestricta de todos los miembros a la obediencia y el cumplimiento de los estatutos. Si bien es cierto es un modelo que separa la política de la administración en busca de la eficiencia, es asimismo un mecanismo que convierte al derecho en una herramienta de poder, un Estado de derecho que sería el reino de las normas, que indica arbitrariamente la realización de lo que debe ser según las reglas ${ }^{10}$.

Esto lo advirtió Claus Offe (1988, p. 11), para quien este principio de la obediencia a la ley toma una significación que se torna en problema, considerando que en el momento en que las normas son creadas solo para lograr los objetivos de la administración pierden su legitimidad, percibiéndose así una predictibilidad y calculabilidad de la actuación burocrática. Ante esta situación, el autor señala la necesidad de un camino alterno que ofrezca una posible fuente de criterios de rectitud en la administración, que al lado de la norma y la adecuación de objetivos lleve a un consenso político.

La respuesta a esta necesidad es aportada parcialmente por Hans Kelsen (1934), quien delimita el Estado de derecho propuesto por Weber, al establecer que las normas están concebidas para la protección de los derechos de los ciudadanos; determina así que en ese conjunto en donde ocurren todos los fenómenos sociales como es el Estado, se debe controlar que los más fuertes no establezcan su dominio y conviertan al Estado en una relación de fuerzas políticas, dentro de la que no está desagregada la administración. En este sentido, el Estado pasa a ser un símbolo del principio altruista-universal, y la sociedad una representación del individualismo egoísta que debe ser controlada, considerando así a la sociedad en un sentido hobbsiano como una conexión de causas y efectos producto de la naturaleza humana, en la que debe obrar el derecho para indicar el deber ser. De esta forma se construye un concepto antagonista entre el Estado (deber ser) y la sociedad, un sistema normativo que no tiene en cuenta al ser humano en su totalidad. Acotando en consecuencia Chevalier (2015, p. 183), "que el reino exclusivo de las normas tiende a extinguir la dinámica social y política socavando los fundamentos mismos del Estado de derecho".

9 Véase en Economía y sociedad el capítulo Ix, Sociología de la dominación (Weber, 1964, pp. 695-1024).

10 Hay que mencionar que Weber, en su libro Economía y sociedad (1964, pp. 498-682), realiza una exegesis del nacimiento y la formación del derecho, en la que enuncia a la obediencia como deber moral, sin embargo, el enfoque de su teoría es el de la centralidad de la administración y la obediencia irrestricta a la norma. 
Las teorías esbozadas, sin lugar a dudas, han hecho aportes significativos para la comprensión del Estado, no obstante, dejan vacíos al no tener en cuenta la integralidad del ser humano; asimismo, su complejidad hace igualmente difícil enmarcar su comportamiento y la manera de moldearlo. Es por ello que se considera que dentro de estas construcciones académicas se trató a las personas como seres autómatas que se deben disciplinar y someter a un régimen de derecho. Tampoco ha sido solo la coerción y la guerra la que ha llevado al ser humano a fijarse objetivos comunes, esto está enmarcado dentro de un exclusivo actuar racional y obvia los sentimientos morales que edifican las sociedades.

\section{RAZONES POR LAS CUALES EL ESTADO REQUIERE DEL RECONOCIMIENTO PARA SU CONSOLIDACIÓN}

Ahora se hace necesario traer a colación diferentes reflexiones de algunos autores que, tendiendo al positivismo y al predominio del derecho, dejan de lado las particularidades emotivas del ser humano, para cotejarlas con valiosos aportes académicos que exaltan la espontaneidad del individuo sobre la imposición restrictiva o norma negativa. Esta reflexión conduce a plantear que el ambiente determina el actuar del individuo, estableciendo en su estructura mental unas instituciones informales que pasan a determinar las formas de asociación y los principios que conforman la convivencia.

La modernidad, con su novedad antropológica, trae consigo el conflicto entre lo normativo y el realismo, entre el buen actuar moral y el comportamiento egoísta que busca un interés particular. No ha sido fácil para el ser humano aceptar que hay unos marcos de acción que no debe transgredir, y que los sentimientos morales deben estar implícitos en su actuar. Es por ello que todas las sociedades han creado un régimen axiológico en el que se exalta el comportamiento virtuoso y se desaprueba o censura el vicioso.

Entre las obras que exaltaron este principio se encuentra la Teoría de los Sentimientos Morales de Adam Smith (2012, pp. 5-27), que en su sección primera enuncia que, sin importar lo egoísta que sea un hombre, hay unos principios en su naturaleza que lo hacen interesarse en la suerte de otros, siendo esto algo necesario para la propia felicidad. Es una suerte de empatía que facilita la interpretación del sufrimiento ajeno y juega un papel importante el provocar sentimientos de aprobación, creando unos estándares por los cuales se llegan a juzgar los propios sentimientos.

El condicionamiento social emocional es relevante en la construcción de convivencia, ella no es solo producto de un actuar racional como lo propone Jellinek (2012, p. 31) al afirmar que todo individuo, por ese hecho positivo de la convivencia y la cooperación, se obliga a guardar las normas para facilitar la existencia (egoísmo inteligente), controlando sus impulsos primarios. Tampoco se limita a restringir la agresividad, como afirma Norbert Elias (2009, p. 283), en el proceso de la civilización, sino que es la exaltación de las convenciones que elogian la virtud, que va de la mano con el principio de aceptación y de reconocimiento 
que reclama cada individuo, lo que realmente establece unas condiciones que hacen posible la vida en comunidad. De ello dimanan coacciones y un control social intenso anclado en la organización estatal que se superpone a las manifestaciones de crueldad, por el cual las acciones violentas se ven limitadas por las sensaciones de desagrado que despiertan.

Este es un proceso evolutivo en el que se van mejorando las condiciones de convivencia, al ir adoptando unos mecanismos sociales que preceden a los disciplinarios que enuncia $\mathrm{Mi}$ chel Foucault (2006, p. 20). Se trata de evitar entrar en el desgaste de castigar al culpable, fijando la medida considerada como óptima y límites de lo aceptable, porque si se transgreden estos límites, sí entran en acción las técnicas policiales que establecieron la división binaria entre lo permitido y lo vedado, haciendo que la opción del transgresor sea poco atractiva a los ojos de los demás mediante una acción del Estado rigurosa y ejemplarizante ${ }^{11}$. De estas dos maneras se previene que se presenten acontecimientos negativos en la convivencia: por una parte está el control social que rechaza las conductas anonímicas, es decir instaura unas instituciones (normas) informales que se caracterizan por no estar tipificadas o registradas en algún documento, sino que son convenciones sociales de obligatorio cumplimiento; y por otro lado está la acción coercitiva del Estado para reprimir la transgresión de las normas debidamente positivizadas (instituciones for- males). Así, las instituciones pasan a ampararse en la lógica de la prioridad del bien común sin obviar la de la individualidad.

En las palabras prácticas de Salvador Rus (2011, p. 105), los seres humanos han organizado su convivencia, planteando un criterio normativo que unifique el proceder en los asuntos públicos y privados que acontecen en un espacio geográfico. Algo que ha ido avanzando en complejidad desde que en aquella lejana polis griega se comenzara a filosofar sobre la mejor forma de construir una sociedad ideal. En este sentido, Hannah Arendt (1993, p. 51) dice que la sociedad espera de cada individuo una cierta clase de conducta mediante la imposición de innumerables y variadas normas, enmarcando así el actuar de sus miembros.

El intervenir en el mundo o abstenerse de hacerlo implica influir en un proceso, es decir convertirse en agente, de esta manera la conducta actúa en la reproducción social. Anthony Giddens (2011, p. 52) hace la salvedad de que esto no se puede interpretar como si los seres humanos fueran autómatas, sino que se debe comprender que los sistemas sociales presuponen relaciones regularizadas con autonomía y dependencia entre colectividades en las que se va conformando la "identidad del yo". La objetividad sin subjetividad, como refiere Hegel (2000, p. 52), es incompleta, y, a la inversa, la subjetividad sin objetividad es vanidad y nulidad. Es la integración de ambos momentos

11 Existen estudios sobre la obediencia al derecho; en primer lugar está la teoría del desincentivo que propone desincentivar conductas a través de las amenazas y la aplicación de castigos o de premios; en segundo lugar esta la teoría normativa que propone el cumplimiento voluntario de las normas a partir de una adherencia moral a estas (Tyler, 2014, pp. 13-14). 
lo que Hegel llama el universal concreto, en donde se construyen las relaciones sociales.

Por consiguiente, se puede interpretar que la razón se ha ido conformando a lo largo de la biografía personal, a partir de continuas elecciones entre valores y sentimientos que se constituyen a través de su relación con otros y su entorno. Hay que tener siempre presente que las emociones en el ser humano juegan un papel importante, siendo ello indispensable para realizar actos morales. Adela Cortina (2009, pp. 80-99) se refiere a los sentimientos como indispensables para alcanzar los estándares de moralidad y los ideales de la humanidad.

Sin lugar a dudas, es la artificialidad humana la que forma y da sentido al mundo, construyendo dentro de un concepto biopolítico un espacio existencial, en el que las personas se caracterizan por su participación en beneficio de la totalidad; esto coincide con lo planteado por Bobbio (1989, p. 66) y Del Águila (2009, p. 15), e indica que los sentimientos preceden a la idea de Hegel de Estado-razón. No se espera solo que el Estado solucione permanentemente los conflictos que existen en la vida cotidiana del hombre, como afirman por ejemplo Bobbio y Bovero (1986, p. 139), sino que los sentimientos morales den testimonio de la naturaleza humana, siendo estos complementados por la razón para mantener la convivencia. De esta manera, el desarrollo de una autoridad contra el predominio de los instintos es precedida por el cumplimiento de las instituciones informales.

Hay que hacer la salvedad de que no se trata de una evolución general del sistema social, sino de mecanismos de evolución pro- pios de cada sociedad, son creaciones parciales como lo indica Víctor Reyes (2016, p. 47). Se trata de pensar cómo los sistemas sociales se autorregulan a través de la construcción de identidades que, aunque siempre serán defectuosas, son una forma de autoprotección efectiva (Solarte, 2016, p. 57).

En este proceso juega un papel importante la autonomía porque ella obliga, tal como lo establece la ética kantiana, debido a que los seres humanos se distinguen por su capacidad de darse leyes a sí mismos, lo que los obliga por ello a cumplirlas. Estas leyes no son arbitrarias, sino aquellas que se consideran dignas de convertirse en leyes de la humanidad. Destaca de esto Adela Cortina (2009, pp. 103-127), que la autonomía tiene dos lados: la capacidad de autoobligarse y la de optar por leyes expresivas de la humanidad. Estas normas llegan a tener autoridad sobre los impulsos, a agudizar la sensibilidad hacia las diferencias y a reconocer la particularidad de las identidades. En consecuencia, el sujeto crea una identidad moral fuente de razones y obligaciones, es decir una norma.

Lo hasta aquí expresado expone claramente que es la acción espontánea la que predomina en el ser humano, son sus emociones lo que en un primer momento lo lleva a actuar, un proceder que conlleva un sentimiento moral que ha asimilado del entorno donde habita, el que implica un reconocimiento del otro y de la norma, formando en consecuencia una comunidad que se organiza con base en la razón. Por este motivo se expone que el Estado tiene como fundamento el reconocimiento para su consolidación. 


\section{LA IMPORTANCIA DE LAS EMOCIONES Y LOS SENTIMIENTOS EN LA CONSOLIDACIÓN DEL ESTADO}

En este momento se inicia una orientación por el psicologismo moral ${ }^{12}$, para explicar cómo cada ser humano moldea y reduce sus pensamientos a través de sus sentimientos, para establecer así los valores morales. De esta manera se puede establecer que, con base en las emociones, las personas determinan en primera instancia sus conductas y sus relaciones con los demás; esto indica que de las emociones depende la cohesión social y, por su conducto, la consolidación del Estado.

Como se ha dicho a lo largo de este texto, las emociones son las que orientan en primera instancia el actuar del ser humano determinando las características de una sociedad. Ellas son las que despiertan el sentimiento moral que lleva a desarrollar la empatía cognitiva que nos permite identificar y comprender las emociones de otras personas, así como la empatía afectiva que se refleja a través de las sensaciones y los sentimientos que tenemos como respuesta a las emociones de los demás. Como son las que determinan en un alto porcentaje el comportamiento del ser humano, se deben tener en cuenta en el desarrollo de las ciencias sociales y, por su conducto, en la construcción de conceptos y teorías de la ciencia política.

Una emoción, dice Daniel Goleman (2011, p. 331) extractando la definición del Oxford English Diccionary, es "cualquier agi- tación y trastorno de la mente, el sentimiento, la pasión; cualquier estado mental vehemente o excitado". Es un término que se utiliza para referirse a un sentimiento y sus pensamientos característicos, a estados psicológicos y biológicos que generan formas de actuar.

Por su relevancia, las emociones han sido ampliamente estudiadas, destacándose entre las indagaciones realizadas las teorías mecanicistas de la emoción, que identifican que la relación entre las emociones y la acción es una conexión causal, algo que Skinner demostró con su investigación científica basada en el condicionamiento de estímulo-respuesta; estos postulados son complementados por las teorías cognitivas que indican que las emociones tienen una conexión con la razón que implica un juicio evaluativo, siendo un tipo de explicación teleológica o intencional (González, 2009, p. 91).

Colíjase por consiguiente que existen unas emociones primarias que ocupan un estado evolutivo primitivo que juegan un papel en la selección natural y adaptación del ser humano, son de carácter más universal y tienen menor dependencia de los factores culturales; por otro lado, las emociones secundarias que surgen de manera posterior dependen con mayor intensidad del contexto social, de normas y de cánones culturales (González, 2009, p. 75).

De esta manera se puede inferir su relevancia en la organización de las colectividades. Ellas, como describe Daniel Goleman (2011, p. 328), son determinantes en la formación

\footnotetext{
12 Psicologismo moral hace referencia a lo que es moldeado y reducido a pensamientos o sentimientos de cada persona; este establece los valores morales a partir de los sentimientos, determina que lo que es moralmente aceptable es lo que mi “yo” determina, centraliza al ser humano en sí mismo eludiendo interpretaciones metafísicas.
} 
del carácter, la autodisciplina, la moral y las costumbres democráticas ${ }^{13}$. De ello depende el desarrollo de una vida virtuosa basada en el autodominio y en la capacidad de motivarse y guiarse, algo que rinde beneficios sociales y es mimetizado o emulado por los demás como lo ha descrito en sus trabajos René Girard ${ }^{14}$. En consecuencia, debe comprenderse que las conductas las programa el cerebro en la práctica del diario vivir y a través de los medios de comunicación ${ }^{15}$. Es una lectura permanente que realiza el cerebro de manera inconsciente, y hace relevante el medio donde se desarrolla el ser humano del que aprende a controlar sus impulsos, sus hábitos y su bien moral.

El ver los eventos desde la perspectiva del otro promueve la tolerancia y la aceptación de la diferencia, algo que en la actualidad es cada vez más requerido en un mundo en constante cambio y vértigo, como lo ha descrito Zygmunt Bauman (2008), que ha nombrado este fenómeno como los "tiempos líquidos". Esto conlleva una constante ampliación de valores según lo enuncia Francesc Torralba (2003), y crea en consecuencia un discurso público que asume compromisos auténticos con los valores cívicos y morales.

Las decisiones que toman las personas son hasta en un $90 \%$ más emocionales, cada emoción ofrece una disposición definida para actuar y para afrontar los retos de la vida.
Acota Daniel Goleman (2011, p. 22) que una visión de la naturaleza humana que pasa por alto el poder de las emociones es miope, los sentimientos cuentan tanto como el pensamiento, incluso es habitual que lo inhiban. Es la respuesta emocional la que actúa primero, a la mente racional le lleva más tiempo; después de un primer momento, a la emoción la acompaña la razón para definir la forma de actuar, es en esos instantes cuando la mente puede controlar el curso de las reacciones. Sin embargo, hay una programación mental que en primera instancia determina el comportamiento de las personas, como lo estableció Thorstein Veblen, llamándole instituciones o "complejo de emulación", que condiciona la forma de actuar de los individuos y, por consiguiente, el dinamismo social.

Al respecto, Martha Nussbaum (2016, p. 166) agrega que las emociones públicas son una fuente de estabilidad para los principios políticos positivos que permite que estos sean efectivos, y ocasiona que las leyes y las instituciones se vuelvan razonablemente justas. Así, las emociones ayudan a sostenerlas e incluso a mejorarlas; de esta manera se puede afirmar que las instituciones encarnan las ideas e intuiciones profundas captadas a través de las emociones.

Así estas emociones regulares en sus expresiones comunican a los otros lo que se siente

\footnotetext{
13 Las costumbres democráticas las podemos definir como el hábito de tomar decisiones con base en el consenso, es el tener siempre presente el querer de los demás.

14 Véase una consolidación de la obra de René Girard en la publicación de Mario Roberto Solarte, Violencia e institución, aportes para una ética de la responsabilidad social (2016, pp. 24-70).

15 Véase en este aspecto, por ejemplo, la obra de Byung-Chul Han (2012; 2014a; 2014b), y la obra de Manuel Castells (2001; 2006).
} 
normalmente de manera involuntaria, sin indicar que su represión y control generen problemas psicológicos como afirmaba la ortodoxia de la teoría posfreudiana, simplemente deben ser canalizadas y procesadas positivamente para que no se tornen tóxicas. Hume (1992, pp. 455-609) expuso que cuando se realizan juicios sobre lo que es correcto o incorrecto desde un punto de vista moral nos dejamos llevar por los sentimientos de aprobación o desaprobación, determinando estos la motivación para actuar correcta o incorrectamente. Entonces, su educación se tornándose trascendental para inculcar las que son aceptadas y legítimas por la sociedad y reprimir las inapropiadas, se trata de una educación axiológica.

Téngase en cuenta que todas las emociones implican un pensamiento o una percepción intencionales dirigidos a un objeto y una valoración realizadas desde un punto de vista personal, atribuyendo una significación en términos de objetivos y fines del agente ${ }^{16}$. Arguye Martha Nussbaum (2016, p. 483) que el contenido cognitivo de las emociones está moldeado por las normas y las circunstancias sociales concretas. Esto destaca que los rasgos generales compartidos de la vida ejercen una notoria influencia en el comportamiento de las personas y desarrollan las circunstancias co- munes de manera diferente en cada sociedad, realizando una taxonomía emocional.

La descripción realizada de las emociones y los sentimientos nos permite apreciar su relevancia en la organización de las comunidades; las emociones son motivadas por los sentimientos que despierta el reconocimiento de la otredad, siendo así un principio relevante para construir sociedad.

\section{EL RECONOCIMIENTO COMO FACTOR RELEVANTE EN LA CONSOLIDACIÓN DEL ESTADO}

El ser humano, en la formulación de teorías políticas, no debe ser tratado como un autómata o como una cosa, es decir con "reificación" 17 , sin tomar en cuenta sus cualidades. La supremacía del modelo sujeto-objeto ${ }^{18}$ afecta la comprensión del ser humano y de su vida en comunidad, de la que hace parte el sentimiento y lo emocional, distanciando la ciencia de la praxis. Es obviar que este es un mundo de sensaciones en el que el aceptar la sensibilidad de otras personas determina al sujeto existencialmente. El proceso de construcción de la identidad está determinado por la mirada y aceptación del otro, este es

\footnotetext{
16 Se entiende por agente una entidad o individuo que incide o influye en la dinámica de la estructura social.

17 Como se mencionó en la nota 8, supra, reificación es un término que Georg Lukács comenzó a utilizar en su colección de ensayos Historia y Conciencia de clase en 1925 (1969, pp. 89-266).

18 El sujeto es el ser humano; fue Fichte (1964) el teórico del sujeto, para él el hombre lo es todo, el yo es la fuente originaria de todo ser cósmico, fíjate en ti mismo, desvía tu mirada de todo lo que te rodea y dirígela a tu interior, el yo cognitivo y el volitivo no tienen límites, elimina la dualidad de sujeto y objeto, el dogmatismo no es filosofía sino una imponente afirmación.
} 
un procedimiento dialógico que conduce al reconocimiento, no es una cortesía sino una necesidad humana vital, que nace de la construcción de identidad dentro de una filosofía de igualdad y dignidad, como bien lo indica Daniel Bonilla (1999, pp. 22-30).

Es el caso, por ejemplo, de la racionalización del Estado y de la sociedad propuesta por Max Weber y otras esbozadas anteriormente, que dan a entender que estas teorías se quedaron atrapadas en el esquema de oposición entre sujeto y objeto. Por ello responde ante esta tendencia Martín Heidegger (2016, p. 32), quien señala que la investigación científica no es determinante cuando explica los posibles modos de ser del ente Dasein ${ }^{19}$, cuya existencialidad está por encima de los demás entes. Por ello, el ser humano y los objetos sociales no deben ser analizados como cosas, sino como seres sensibles retirando el velo óntico detrás del cual se esconde la facticidad de la forma efectiva de la existencia humana.

La referida disolución del individuo obliga a una revisión del conocimiento del sujeto como indica de Theodor Adorno (1998, p. 61), porque el principio de la dominación humana evolucionó hacia un principio absoluto, dejando de lado el yo y su idea rectora que objetiva al ser humano. De esto se percató también John Dewey (1952), quien arguye que los filósofos han menospreciado la acción del obrar y del hacer, y han puesto la teoría sobre la praxis. La invención de las artes puso a disposición del hombre los poderes de la naturaleza, llevando incluso a obviar los sentimientos de las personas; de esta manera materializó y objetivó la ciencia, creando una necesidad por conciliar el conocimiento científico con la validez de las ideas concernientes al valor.

Para Axel Honneth (2007, p. 51), esta línea de pensamiento descrita ha creado una costumbre de pensamiento y perspectiva que se fosilizó, convirtiéndose en hábito adoptar al sujeto sin interés cualitativo. De allí que resulte imperativo que se interprete que la relación del hombre consigo mismo y con el mundo obra con base en una acción de reconocimiento, lo cual debe preceder a cualquier otra definición o conceptualización.

Por consiguiente, no es coherente ni suficiente interpretar la existencia del Estado con base en una motivación racional. El orden político y social de una sociedad, dice Adela Cortina (2009, p. 165), se funda en la exigencia moral de ser reconocido, algo que, como el miedo y el cálculo racional, ha llevado a asociarse. El reconocimiento recíproco crea un vínculo que conlleva la autorreflexión, la conciencia de sí mismo y la orientación hacia el otro. Es la vida ética del reconocimiento recíproco la que constituye la nación-Estado, no solo las abstracciones netamente racionales.

En consecuencia, la valoración cualitativa en que se basa el reconocimiento es fundamental para la explicación de la existencia del ser humano. Es decir, existe una unión emocional o identificación que conduce a la aceptación y valoración de otras perspectivas que se deben

\footnotetext{
19 Dasein, ser-ahí, es la manera como Heidegger nombra al ente hombre en su ideal de ser, su peculiaridad óntica consiste en que es ontológico. Es cada vez su posibilidad, es decir que evoluciona, siempre con la opción de mejorar, de optimizar el ser.
} 
tener en cuenta para comprender el comportamiento de las personas y la forma en que llegan a acuerdos para asociarse. El aprobar el valor del otro está inmerso en la conformación de las naciones y la manera como han llegado a establecer unas instituciones para su coexistencia.

\section{CONCLUSIONES}

Una vez analizadas algunas teorías que han pretendido explicar la existencia del Estado, como la de Thomas Hobbes, y esbozado algunas otras como la de Charles Tilly y la de Max Weber, nos podemos percatar de que sus argumentaciones son muy acertadas y han aportado a la definición conceptual del Estado. No obstante, en ellas se tiende a la reificación del ser humano, obviando que este es un sujeto en el que median constantemente las emociones y los sentimientos que despiertan el anhelo de aliarse con sus semejantes para construir sociedades en las que se sientan identificados para, con base en alianzas y acuerdos morales, hacer frente al miedo que representa la incertidumbre.

La idea de Thomas Hobbes de que el Estado surge para mitigar la acción voluntaria natural de las personas, y así hacer posible la convivencia, es complementada por Adela Cortina quien aduce que ese egoísmo inteligente es el resorte que mueve a las personas a ampliar las leyes legales pero no para adquirir convicciones morales, por tanto, surge la necesidad de hacer de la obligación legal también una obligación moral, al ser justo y convenir a la generalidad.
En cuanto al poder absoluto expuesto por Hobbes, aduce Cortina, es solo exterior, no convence las mentes, no vincula a las personas consigo mismas y con las otras, no genera obligación moral, ignora el aprecio por lo valioso, el disfrute de la relación mutua, la estima de la dignidad ajena y la propia, que es lo que realmente construye y crea comunidad.

En observación a la fundamentación del individualismo posesivo de Hobbes, Cortina responde aduciendo que el individualismo depende inevitablemente del "principio del intercambio infinito", al ser los seres humanos individuos de carencias y, por ello, necesitan de otras personas y de lo que el entorno ambiental ofrezca. El intercambio y la cooperación son más inteligentes que la fuerza bruta, esta lógica depende de la empatía que incluye los sentimientos sociales.

Al hacer referencia a la teoría clásica, tradicional y ortodoxa de Max Weber, que se fundamenta en la organización burocrática, racional y unitaria donde predomina el poder de la rama ejecutiva, da a entender que todo ejercicio de autoridad es un ejercicio de administración, que convierte al derecho en una herramienta de poder, un Estado de derecho que sería el reino de las normas que indican el deber ser, de donde se colige que estas se hicieron para satisfacer los objetivos de la administración y perdieron su legitimidad. Cortina responde que el motivo originario del establecimiento de la justicia se causa en la simpatía por el interés público como fuente de aprobación moral que elogia la virtud, permitiendo así el transitar de la obligación natural a la obligación moral, por consiguiente son los sentimientos sociales 
los que mantienen moralmente a la sociedad cohesionada, no la imposición.

Esto nos lleva a determinar que las emociones y los sentimientos son los causantes de que se despierten empatías y antipatías en el obrar de las personas, desarrollando juicios de valor en cada acto de vida. Es decir, son ellas las que motivan los sentimientos morales que conducen al buen actuar y orientan a las personas a asociarse con individuos que identifiquen la misma necesidad de justicia y acepten, con base en ella, asumir unas pautas de comportamiento o normas. Las emociones son las que en definitiva inducen a las personas a actuar; después de una primera reacción entra la conciencia a obrar, sin embargo, las decisiones que se asuman irán siempre determinadas por un alto porcentaje emocional. Esto nos da a entender que los sentimientos y las emociones humanas han hecho parte de la consolidación de la sociedad y del Estado.

Es necesario entender que las emociones se fundamentan en el sentimiento que despierta la otredad, es decir, son una acción de reconocimiento. Son una construcción de conocimiento que se desarrolla con base en el sentimiento que gesta la unión, como una representación de aceptación de la individualidad con sus particularidades, en las que van inmersas sus sensaciones subjetivas conformando en consecuencia una multiplicación de significaciones que son relevantes para la construcción de la vida en comunidad; de su reconocimiento depende que se conformen sociedades, en un proceso en que es indispensable iniciar por el reconocimiento personal para después pasar al de los demás y así llegar a acuerdos sólidos que permitan la consolidación del Estado.

\section{REFERENCIAS}

Adorno, T. (1998). Minima moralia. Madrid: Taurus. Agamben, G. (2017). Stasis, La guerra civil como paradigma politico. Buenos Aires: Adriana Hidalgo.

Allendesalazar, M. (1988). Spinoza, filosofía, pasiones y politica. Madrid : Alianza.

Arendt, H. (1993). La condición humana. Barcelona: Paidós.

Bauman, Z. (2008). Tiempos líquidos, vivir en una época de la incertidumbre. México D.F.: Tusquets.

Bobbio, N. (1989). Estado, gobierno y sociedad, por una teoría general de la politica. México: Fondo de Cultura Económica.

Bobbio, N. y Bovero, M. (1986). Sociedad y Estado en la filosofia moderna, el modelo iusnaturalista y el modelo hegeliano-marxiano. Bogotá: Fondo de Cultura Económica.

Bonilla, D. (1999). La ciudadanía multicultural y la politica del reconocimiento. Bogotá: Universidad de los Andes.

Buchely, L. (2014). Las burocracias. Bogotá: Siglo del Hombre.

Castells, M. (2001). La galaxia internet. Barcelona: Plaza y Janés.

Castells, M. (2006). La sociedad red: una visión global. Madrid: Alianza.

Chevallier, J. (2011). El Estado posmoderno. Bogotá: Universidad Externado de Colombia.

Cortina, A. (2009). Ética de la razón cordial, educar en la ciudadanía en el siglo XXI. Madrid: Nobel.

Del Águila, R. (2009). La inclasificable teoría política de Hannah Arendt: poder, acción y juicio. En R. Máiz, Teorias politicas contemporáneas (pp. 13-26). Valencia: Tirant lo Blanch.

Dewey, J. (1952). La busca de la certeza. México: Fondo de Cultura Económica. 
Duso, G. (2005). Elpoder, para una historia de la filosofia politica moderna. México: Siglo xxi.

Elias, N. (2009). El proceso de la civilización, investigaciones sociogenéticas y psicogenéticas. México: Fondo de Cultura Económica.

Fichte, J. (1964). Primera y segunda introducción a la teoría de la ciencia. México: Fondo de Cultura Económica.

Foucault, M. (2006). Seguridad, territorio, población. México: Fondo de Cultura Económica.

Foucault, M. (2016). Nacimiento de la biopolitica. Buenos Aires: Fondo de Cultura Económica.

Giddens, A. (2011). La construcción de la sociedad, bases para la teoría de la estructuración. Buenos Aires: Amorrortu.

Goleman, D. (2011). La inteligencia emocional, por qué es más importante que el cociente intelectual. México D.F.: Zeta.

González, D. (2009). Emociones, responsabilidad y derecho. Madrid: Marcial Pons.

Han, B. (2012). La sociedad del cansancio. Barcelona: Herder.

Han, B. (2014a). Psicopolitica. Barcelona: Herder.

Han, B. (2014b). En el enjambre. Barcelona: Herder.

Hegel, G. (2000). Rasgos fundamentales de la filosofia del derecho o compendio de derecho natural y ciencia del Estado. Madrid: Biblioteca Nueva.

Hegel, G. (2009). Fenomenología del espiritu. Valencia: Pre-textos.

Heidegger, M. (2016). Ser y tiempo. Madrid: Trotta.

Hobbes, T. (1980). Leviatan. México D.C.: Fondo de Cultura Económica.

Honneth, A. (2007). Reificación, un estudio en la teoría del reconocimiento. Buenos Aires: Katz.

Hume, D. (1992). Tratado de la naturaleza humana. Madrid: Tecnos.
Jellinek, G. (2012). Teoría general del Estado. México: Fondo de Cultura Económica.

Kaldor, M. (2010). El poder y la fuerza, la seguridad de la población civil en un mundo global. Barcelona: Tusquets.

Kelsen, H. (1934). Teoría general del Estado. Madrid: Labor.

Londoño, F. (2011). Esbozo de una teoría general de la ciencia de policía. Bogotá: Policía Nacional de Colombia.

Lukács, G. (1969). Historia y conciencia de clase. México: Grijalbo.

MacPherson, C. (2005). La teoría politica del individualismo posesivo, de Hobbes a Locke. Madrid: Trotta.

North, D. (1993). Instituciones, cambio institucional y desempeño económico. México: Fondo de Cultura Económica.

Nussbaum, M. (2016). Emociones politicas ¿Por qué el amor es importante para la justicia? Bogotá: Paidós.

Offe, C. (1988). Partidos politicos y nuevos movimientos sociales. Madrid: Sistema.

Reyes, V. (2016). La anomia, espacios, tiempos y conflictos anómicos, análisis de casos. Bogotá: Aurora.

Rus, S. (2011). Aristóteles: la política como dimensión y exigencia del ser humano. En P. Sánchez y Martínez, C. Historia del análisis politico (pp. 93-126). Madrid: Tecnos.

Smith, A. (2012). Teoría de los sentimientos morales. Medellín: Universidad de Antioquia.

Solarte, M. (2016). Violencia e institución, aportes para una ética de la responsabilidad social. Bogotá: Universidad Javeriana.

Spinoza, B. (1986). Tratado teológico y político. Madrid: Alianza.

Spinoza, B. (1986). Tratado politico. Madrid: Alianza. 
Tilly, C. (1992). Coerción, capital y los Estados europeos 990-1990. Madrid: Alianza.

Torralba, F. (2003). Cien valores para una vida plena, la persona y su acción en el mundo. Madrid: Milenio. Universidad Nacional Autónoma de México.

Tyler, T. (2014). La obediencia del derecho. Bogotá D.C: Siglo del Hombre.

Vela, B. (2010). Contribución al debate sobre la formación del Estado colombiano en el siglo XIX. Bogotá: Universidad Externado de Colombia.

Vela, B. (2011). La economía de Europa hasta antes de la caída del muro de Berlín: ¿guerras por la economía o economías de guerra? En B. Vela, Lecciones sobre Europa (pp. 69-96). Bogotá: Universidad Externado de Colombia.
Wallerstein, I. (2006). El moderno sistema mundial, la segunda era de gran expansión de la economiamundo capitalista 1730-1850. México: Siglo XXI.

Weber, M. (1964). Economía y sociedad, esbozo de sociología comprensiva. México: Fondo de Cultura Económica.

Villoria, M. e Izquierdo, A. (2016). Ética pública y buen gobierno, regenerando la democracia y luchando contra la corrupción desde el servicio público. Madrid: Tecnos. 